\title{
Validade concorrente do peso e estatura auto-referidos no diagnóstico do estado nutricional em mulheres idosas
}

\section{Concurrent validity of self-reported weight and height for diagnosing elderly women's nutritional status}

\author{
Jair Sindra Virtuoso-Júnior ${ }^{1}$ y Ricardo Oliveira-Guerra ${ }^{2}$ \\ 1 Instituto de Ciências da Saúde, Universidade Federal do Triângulo Mineiro. Uberaba, MG, Brasil. \\ virtuosojr@yahoo.com.br \\ 2 Departamento de Fisioterapia, Universidade Federal do Rio Grande do Norte. Natal, RN, Brasil.
}

Recebido 29 Maios 2009/Enviado para Modificação 31 Dezembro 2009/Aprovado 17 Janeiro 2010

\section{RESUMO}

Objetivo Analisar a validade concorrente de medidas auto-referidas de peso e estatura para o diagnóstico do estado nutricional em mulheres idosas de baixa renda.

Métodos $\mathrm{A}$ amostra foi composta de 181 mulheres, com média de idade de 70 $\pm 7,2$ anos, pertencente a grupos de atividades para idosos na comunidade no município de Jequié, Bahia. As medidas referidas de peso e estatura corporal foram coletadas através de entrevista individual que antecederam as aferições. $\mathrm{Na}$ análise dos dados foram utilizadas medidas de tendência central e dispersão para caracterização da amostra, utilizou-se o test t Student para avaliar as diferenças entre as médias das medidas referidas com as aferidas; o coeficiente de correlação intraclasse e o índice Kappa foram utilizados para verificação da concordância entre as medidas; $p<0,05$.

Resultados Os resultados indicam haver uma tendência para a subestimação das medidas antropométricas de peso e estatura referidas. Foram observados índices satisfatórios de concordância para as medidas referidas de peso corporal variando de $r_{\text {icc }}=0,74$ em pessoas eutróficas, até $r_{\text {icc }}=0,97$ considerando as variáveis, classe econômica e ausência de déficit cognitivo. Porém, em medidas referidas de altura os índices de concordância foram baixos variando de $r_{\text {icc }}=0,03$ a $r_{\text {icc }}=0,28$.

Conclusões Os dados analisados permitem concluir que em populações idosas de comunidades de baixa renda e escolaridade, as medidas referidas de peso corporal podem ser consideradas de validade assegurada. No entanto, o mesmo não se pode afirmar das medidas reportadas de estatura, assim como do indicador do estado nutricional baseado em medidas referidas de peso e estatura conjugados.

Palavras Chave: Validade dos testes, antropometria, estado nutricional, idoso (fonte: DeCS, BIREME). 


\begin{abstract}
Objective The present study was aimed at assessing the concurrent validity of selfreported weight and height measurements when diagnosing low-income elderly women's nutritional state.

Methods The sample consisted of 181 women (average age being 70 \pm 7.2 ) taken from groups running activities for elderly people in the city of Jequié, Bahia, Brazil. Reported weight and height measurements were collected through individual interviews preceding direct measurements being taken. Central tendency and dispersion measurements were used during data analysis for characterising the sample and Student's t-test was used for evaluating the differences between the averages for the reported and real measurements. The intraclass correlation coefficient and the Kappa index were used for checking agreement between measurements $(p<0.05)$.

Results The results highlighted a tendency to underestimate reported weight and height anthropometric measurements. Satisfactory agreement indexes were noted for the reported weight measurements (varying from $r_{\text {icc }}=0.74$ to $r_{\text {icc }}=0.97$ in eutrophic people) when considering economic class and the absence of cognitive deficit. On the other hand, concordance indexes for the reported height measurements were low (varying from $r_{\text {icc }}=0.03$ to 0.28 ).

Conclusions Data analysis results led to concluding that the reported weight measurements could be considered to be valid in elderly, low-income, low scholastic level populations. However, the same cannot be said about the reported height measurements, or about the use of the nutritional state indicator, based on combined reported weight and height measurements.
\end{abstract}

Key Words: Validity, anthropometry, nutrition status, the elderly (source: $M e S H, N L M$ ).

\title{
RESUMEN
}

Validez de las medidas auto-referidas y combinadas de peso y altura en el diagnostico del estado nutricional en mujeres de edad avanzada

Objetivo El objetivo del estudio fue evaluar la validez de las medidas auto-referidas de peso y la altura combinadas para el diagnóstico del estado nutricional en mujeres de edad avanzada con bajos ingresos.

Métodos La muestra consistió de 181 mujeres, con una edad media de 70 $\pm 7,2$ años, pertenecientes a grupos de actividades para personas mayores en la comunidad, en el municipio de Jequié, Bahia. Las medidas auto-reporte de peso corporal y la altura fueron obtenidas por medio de entrevistas individuales que precedieron las mediciones directas. En el análisis de los datos se utilizaron medidas de tendencia central y dispersión para caracterizar la muestra, la prueba t de Student para evaluar diferencias entre medias; el coeficiente de correlación intraclase y el índice Kappa fueron utilizados para comprobar la concordancia entre las medidas, con un $\mathrm{p}<0,05$.

Resultados Los resultados indican que hay una tendencia a subestimar las medidas antropométricas de peso y altura reportadas. Se encontraron índices satisfactorios de concordancia en las medidas de peso corporal, con una variación de $R_{\text {icc }}=0,74$ en personas eutróficas hasta $R_{\text {icc }}=0,97$, considerando los aspectos de clase económica y déficit cognitivo. Sin embargo, medidas de altura e índices de concordancia fueran bajos con la variación de $R_{\text {icc }}=0,03$ hasta $R_{\text {icc }}=0,28$. 
Conclusiones Los datos analizados muestran que en la población de ancianos en las comunidades de bajos ingresos y educación, las medidas de peso corporal pueden considerarse validas. Sin embargo, lo mismo no puede afirmarse de las medidas de estatura, ni en cuanto al indicador de estado nutricional, con base en medidas de peso y altura combinadas.

Palabras Clave: Validez de las prueba, antropometría, estado nutricional, anciano (fuente: DeCS, BIREME).

\footnotetext{
medida do peso corporal é um importante indicador na avaliação do 1 estado nutricional das pessoas, principalmente quando conjugada com 1 outras medidas antropométricas, a exemplo da estatura e determinadas circunferências corpóreas. Entretanto, para a viabilidade na avaliação de variáveis antropométricas em levantamentos populacionais, as medidas diretas, na maior parte das vezes, são substituídas por medidas referidas.
}

Há estudos destacando a confiabilidade de medidas antropométricas referidas (1-3). No entanto, a maior parte desses estudos foram realizados em populações jovens, em que o fator preponderante nessa faixa etária é a estética. Tal fato parece favorecer para que as pessoas periodicamente sejam auto-monitoradas em relação ao peso corporal e consequentemente, minimizar as possíveis distorções resultantes das medidas auto-reportadas de peso corporal.

Em pessoas mais velhas, em que os inquéritos populacionais são realizados quase sempre com base na localização domiciliar, as medidas antropométricas aferidas se tornam mais difíceis do que comparada à avaliação de outros grupos etários. E ainda, alguns fatores da senescência como mudanças corpóreas (4) e diminuição da função cognitiva $(5,6)$ faz com que se tenha mais atenção na qualidade das medidas reportadas por pessoas idosas.

Os dados apresentados pelo Instituto Brasileiro de Geografia e Estatística, no que tange a proporção de idosos por gênero evidenciam uma expressiva predominância das mulheres sobre os homens no Brasil (7). Sendo que as mulheres quando comparadas aos homens da mesma faixa tendem a subestimar o peso (8) e superestimar a estatura corporal (1).

Com base no exposto, o propósito dessa investigação foi de analisar a validade concorrente das medidas auto-referidas de peso e estatura para diagnóstico do estado nutricional de mulheres idosas de baixa renda e escolaridade. 


\section{MÉTODO}

Foi realizado um estudo transversal no município de Jequié, localizada 365 km de Salvador, capital da Bahia, Região Nordeste do Brasil, no período de outubro a dezembro de 2006. A amostra final de 181 mulheres idosas com 60 anos ou mais foi selecionada de forma probabilística e estratificada por grupo de convivência. $O$ valor da amostra foi proporcional a $42,3 \%$ da população inicial de 528 idosos que representa o número total de sujeitos participantes nos 16 grupos de convivência do município de Jequié, estabelecendo-se nível de confiança de $95 \%$ com erro de $5 \%$.

A coleta de dados foi realizada por quatro profissionais de Educação Física e de Fisioterapia devidamente treinados, por intermédio de palestras e aplicação dos instrumentos. Para a avaliação foi elaborada uma entrevista multidimensional que foi aplicada de forma individual contendo informações sócio-demográficas (idade, estado civil, classificação econômica (9), escolaridade) e relacionadas à saúde (prática de atividade física, déficit cognitivo (10) e sintomatologia depressiva (11)) para caracterização da amostra, além das medidas referidas de peso e estatura corporal.

Na classificação econômica foi utilizada o Critério de Classificação Econômica Brasil (9) com a finalidade de estimar o poder de compras das pessoas e famílias urbanas, e com isso obter uma distinção em sete classes econômicas $\left(\mathrm{A}_{1}, \mathrm{~A}_{2}, \mathrm{~B}_{1}, \mathrm{~B}_{2}, \mathrm{C}, \mathrm{D}, \mathrm{E}\right)$.

As medidas referidas de peso e estatura corporal precediam as medidas aferidas, no intuito de evitar interferência na percepção da avaliada As idosas foram pesadas e medidas descalças e com roupas leves. A medida do peso corporal real foi realizada com o auxílio de uma balança digital de marca Plenna, modelo Sport, com capacidade para $150 \mathrm{Kg}$ e precisão de 100 gramas. Para a medida da estatura foi utilizado um estadiômetro portátil de marca Seca, com precisão de $0,1 \mathrm{~cm}$.

O Índice de Massa Corporal (IMC) foi calculado dividindo-se o peso $(\mathrm{Kg})$ pela estatura $(\mathrm{m})$ ao quadrado. Na classificação do estado nutricional pelos valores de IMC foram utilizadas as recomendações da Organização Mundial da Saúde (12): $<18,5 \mathrm{Kg} / \mathrm{m}^{2}$ (baixo peso); 18,5 a 24,99 Kg/m² (peso normal); 25 a $29,99 \mathrm{Kg} / \mathrm{m}^{2}$ (sobrepeso) e $30 \mathrm{Kg} / \mathrm{m}^{2}$ (obesidade). O termo utilizado " medidas referidas de IMC" se refere a medida conjugada de peso e da altura referidas. 
Para confecção do banco de dados, foi utilizado o software Epidata, versão $3.1 b$, e as análises por meio do pacote estatístico SPSS (versão 10.0). Na análise descritiva foi feita a distribuição de frequiências absolutas, a distribuição percentual, média e desvio padrão ( \pm ). Precedendo a inferência dos dados foram identificados os valores faltosos e, os mesmos (41 casos), foram excluídos da planilha de dados. Esses casos correspondem aos indivíduos sem informação sobre a estatura referida (38 casos) e aquelas sem informação sobre o peso corporal referido (3 casos). O elevado número de pessoas que deixaram de responder a questão sobre a estatura referida parece ser um indicativo prévio do estudo, das limitações para se obter informações sobre a estatura em pessoas idosas de baixa renda e escolaridade.

$\mathrm{Na}$ abordagem analítica o teste $t$ de Student pareado foi utilizado para avaliar as diferenças entre as médias das medidas referidas e aferidas segundo as categorias de idade (60-69; 70-79-80-92), escolaridade ( $<2$ anos; $\geq 2$ anos); IMC ( $<18,5 ; 18,5-24,99 ; 25-29,99 ; \geq 30)$; classe econômica (A e B; C; D eE); déficit cognitivo (presença/ausência) e participação em atividades físicas de lazer.

Os erros na informação da estatura, do peso e do IMC foram obtidos pela diferença entre os valores referidos e aferidos. Os valores negativos significaram subestimação e valores positivos representaram superestimação do valor referido.

Para avaliação da concordância entre as variáveis de razão, no que se refere as medidas aferidas e referidas de peso, estatura e IMC foi utilizado o coeficiente de correlação intraclasse $\left(\mathrm{r}_{\text {icc }}\right)$. A concordância entre as variáveis ordinais, relativas ao IMC foi analisada por intermédio do Índice Kappa (K).

Para interpretação dos dados analisados foi considerado um nível de significância $p<0,05$ e estimados intervalos de $95 \%$ de confiança. Consideraramse os critérios de Landis e Kock (13) para interpretação da concordância: a) quase perfeita: 0,80 a 1,00 ; b) substancial: 0,60 a 0,80 ; c) moderada: 0,40 a 0,$60 ;$ d) regular: 0,20 a 0,$40 ;$ d) discreta: 0 a 0,$20 ;$ e) pobre: $-1,00$ a 0.

Esta pesquisa seguiu os princípios éticos presentes na Declaração de Helsinque ena Resolução 196/96 do Conselho Nacional de Saúde. Os protocolos de pesquisa foram avaliados e aprovados pelo Comitê de Ética em Pesquisa com Seres Humanos da Universidade Estadual do Sudoeste da Bahia (Parecer n ${ }^{\circ}$ 155/06). 


\section{RESULTADOS}

A amostra final do estudo constituiu-se de 181 mulheres, com a média de idade de $68 \pm 6,6$ anos, com a amplitude de 60 a 92 anos. Embora, tenham sido observadas algumas variações nos grupos etários estabelecidos, a população em geral pode ser descrita com predominância de viúvas 45,3\% $(\mathrm{n}=82)$; o arranjo familiar caracterizado como sendo multigeracional $33,7 \%$ $(\mathrm{n}=61)$; sendo de baixo nível de escolaridade, em que as idosas que referiram ser analfabetas representavam $34 \%(n=64)$; e com $69 \%(n=125)$ das participantes classificadas nas classes econômicas D e E.

O estado nutricional verificado pelo IMC demonstrou que $62 \%(\mathrm{n}=129)$ estavam classificadas na condição de sobrepeso ou obesidade (IMC superior a $24,9 \mathrm{Kg} / \mathrm{m} 2)$. Os sintomas depressivos foram relatados por $18,8 \%(\mathrm{n}=34)$ das participantes, sendo que a alteração do estado cognitivo foi identificada em $72,4 \%$ (n=131). Em relação ao momento de lazer 48,6 \% (n=88) referiram que não participa de práticas de atividades físicas no presente.

As mulheres tenderam a subestimar o peso, porém, sem significância estatística (Tabela 1). A análise das diferenças, segundo as categorias das variáveis estudadas, mostra que a subestimação do peso foi significativa para as mulheres nas faixas etárias de 60 a 69 anos e de 70 a 79 anos, para aquelas com menos de dois anos de escolaridade, para os de classe econômica $\mathrm{C}$, com o IMC igual ou superior a $25 \mathrm{Kg} / \mathrm{m}^{2}$ e para aquelas que participam de atividades físicas no lazer. No entanto, o déficit cognitivo não foi identificado diferenças entre as categorias estabelecidas (presença ou ausência de alterações cognitivas), ambas as categorias foram identificadas diferenças significativas entre os valores aferidos com os referidos para o peso corporal.

Em relação às diferenças entre as medidas aferidas e referidas de estatura e do IMC, pode se verificar que as idosas tenderam a subestimar as medidas, porém somente as categorias de IMC classificadas como "normal" ou "obesidade" se mostram significativas para estatura. Para o IMC também foram evidenciadas diferenças significativas para as mesmas variáveis, acrescidas da ausência do déficit cognitivo. Porém as que estavam classificadas como "normal" tenderam a subestimar os valores referidos ao contrário das que estava na categoria "obesidade" que tendem a superestimar. 
Tabela 1. Diferença e coeficiente de correlação intraclasse $\left(r_{\text {icc }}\right)$ entre as medidas referidas e aferidas de peso, altura e IMC em mulheres segundo idade, variáveis socioeconômicas e antropométricas

\begin{tabular}{|c|c|c|c|c|c|c|c|}
\hline \multirow{2}{*}{ Variáveis } & \multirow[t]{2}{*}{$\mathrm{N}$} & \multicolumn{2}{|c|}{ Diferença no peso } & \multicolumn{2}{|c|}{ Diferença na altura } & \multicolumn{2}{|c|}{ Diferença no IMC } \\
\hline & & Média (tDP) & $r_{i o s}$ & Média $(t D P)$ & $r_{i 00}$ & Média $( \pm D P)$ & $r_{i c 0}$ \\
\hline Total & 181 & $-1,30(5,5)^{\star *}$ & 0,90 & $-1,82(20,4)^{*}$ & 0,18 & $7,18(0,94)^{*}$ & 0,44 \\
\hline \multicolumn{8}{|l|}{ Faixa etária (anos) } \\
\hline $60-69$ & 95 & $-1,45(5,7)^{\star \star}$ & 0,90 & $-2,28(20,6)^{\star}$ & 0,24 & $0,12(0,86)^{\star}$ & 0,52 \\
\hline $70-79$ & 70 & $-1,14(4,5)^{\star *}$ & 0,91 & $-1,22(18,9)^{*}$ & $0,08^{*}$ & $1,14(0,91)^{*}$ & 0,44 \\
\hline $80-96$ & 16 & $-1,06(8,3)^{\star}$ & 0,79 & $-1,72(26,0)^{\star}$ & $0,12^{*}$ & $6,25(1,44)^{*}$ & $0,10^{*}$ \\
\hline \multicolumn{8}{|l|}{ Escolaridade } \\
\hline$<2$ anos & 88 & $-2,18(6,7)^{\star \star}$ & 0,85 & $-4,32(24,7)^{\star}$ & $0,13^{\star}$ & $2,2(1,09)^{\star}$ & 0,28 \\
\hline$\geq 2$ anos & 93 & $-0,47(3,9)^{\star}$ & 0,94 & $0,54(15,0)^{*}$ & 0,26 & $0,12(0,76)^{*}$ & 0,61 \\
\hline \multicolumn{8}{|l|}{ Classe Econômica } \\
\hline$A, B$ & 10 & $0,53(3,18)^{\star}$ & 0,94 & $-6,80(23,0)^{\star}$ & $0,25^{\star}$ & $0,00(0,94)^{*}$ & $0,21^{*}$ \\
\hline C & 46 & $-1,18(2,5)^{\star *}$ & 0,97 & $0,88(14,1)^{\star}$ & $0,28^{*}$ & $0,20(0,81)^{*}$ & 0,51 \\
\hline $\mathrm{D}, \mathrm{E}$ & 125 & $-1,49(6,4)^{\star}$ & 0,87 & $-2,42(22,1)^{\star}$ & $0,22^{*}$ & $3,20(0,98)^{*}$ & 0,43 \\
\hline \multicolumn{8}{|l|}{ IMC $\left(\mathrm{Kg} / \mathrm{m}^{2}\right)$} \\
\hline$<18,5$ & 3 & $-3,66(18,5)^{\star}$ & 0,80 & $-21,7(32,1)^{\star}$ & $0,03^{*}$ & $-1,00(1,73)^{\star}$ & $0,00^{*}$ \\
\hline $18,5-24,99$ & 56 & $-0,51(6,5)^{\star}$ & 0,74 & $-7,2(21,9)^{\star \star}$ & $0,20^{*}$ & $-0,4(0,93)^{\star *}$ & $0,00^{*}$ \\
\hline $25-29,99$ & 65 & $-1,56(4,2)^{\star *}$ & 0,83 & $-8,15(2,56)^{*}$ & $0,20^{*}$ & $0,11(0,85)^{*}$ & $0,00^{*}$ \\
\hline$\geq 30$ & 57 & $-1,65(4,8)^{\star \star}$ & 0,94 & $2,58(16,3)^{\star *}$ & $0,20^{*}$ & $0,56(0,71)^{\star \star}$ & $0,00^{*}$ \\
\hline \multicolumn{8}{|l|}{ Déficit Cognitivo } \\
\hline Ausência & 50 & $-1,24(3,1)^{\star *}$ & 0,97 & $-1,55(18,5)^{*}$ & $0,27^{*}$ & $0,22(0,74)^{\star *}$ & 0,67 \\
\hline Presença & 88 & $-1,32(6,2)^{\star *}$ & 0,85 & $-2,89(22,3)^{*}$ & $0,15^{\star}$ & $1,53(1,00)^{*}$ & 0,35 \\
\hline \multicolumn{8}{|l|}{ Atividade Física } \\
\hline Participa & 93 & $-1,79(5,9)^{* *}$ & 0,88 & $-1,55(18,5)^{*}$ & 0,27 & $5,3(0,90)^{*}$ & 0,47 \\
\hline Não participa & 88 & $-0,78(5,0)^{*}$ & 0,91 & $-2,1(22,3)^{*}$ & $0,09^{*}$ & $9,0(0,98)^{*}$ & 0,42 \\
\hline
\end{tabular}

${ }^{*}$ Diferença $=$ valor referido menos valor aferido; ${ }^{*}$ Média de diferenças e desvio padrão $(p<0,05$ no teste t de Student pareado)

Os coeficientes de correlação intraclasse entre as medidas do peso reportadas e aferidas foram de 0,79 a 0,94 , índices classificados como concordância "substancial" para "quase perfeita". No entanto, os valores correspondentes ao grau de concordância entre as medidas aferidas com as reportadas para a estatura e IMC foram menores, sendo classificadas de uma concordância "pobre" a "regular" (Tabela 1).

Ao considerar o IMC aferido como padrão, observa-se que 33,3 \% das idosas de baixo peso foram classificadas como normais, utilizando-se o IMC referido. No outro extremo, 36,9\% foram classificadas com sobrepeso, $7 \%$ como normais e 1,7 \% com baixo peso. Apesar das variações entre os valores reportados e aferidos, o IMC reportado possui uma concordância regular com as medidas aferidas (Figura 1). 
Figura 1. Distribuição das idosas segundo as categorias de classificação do IMC aferido e reportado

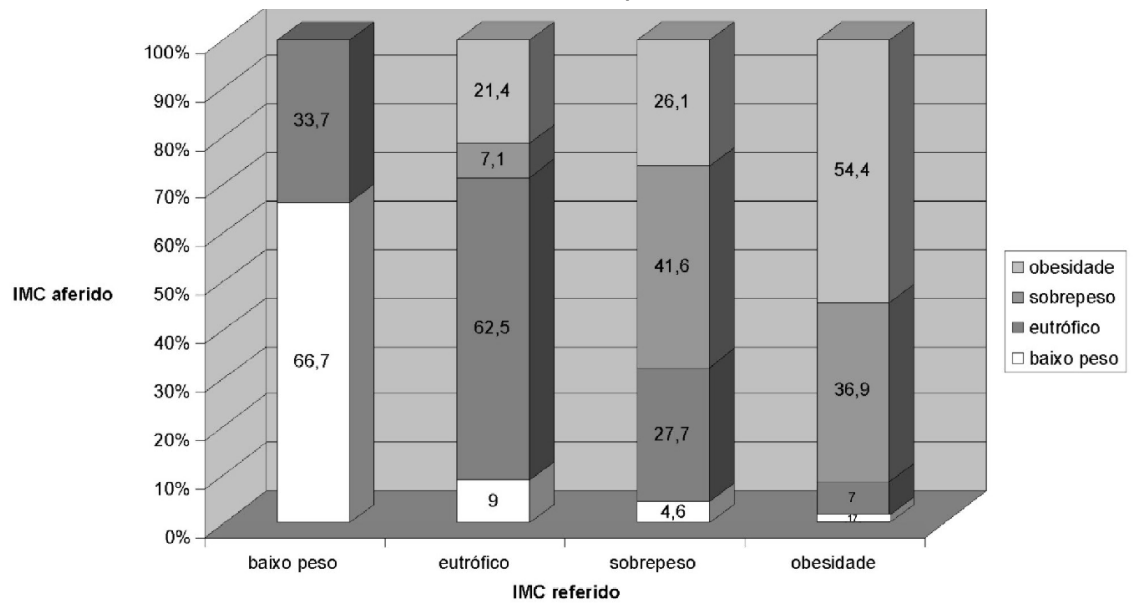

Com isto, observa-se para as idosas, a concordância na identificação do baixo peso e normal foi de $66,7 \%$ e $62,5 \%$ respectivamente, sendo menor $(41,6 \%)$ na identificação do sobrepeso (Figura 1).

\section{DISCUSSÃO}

O aumento das doenças crônicas não transmissíveis tem feito que haja um maior interesse em estudos que possam identificar fatores que estejam associadas às condições de saúde das pessoas. Nesses estudos, baseados em levantamentos populacionais, as medidas reportadas são utilizadas com frequiência, em virtude da viabilidade, em função principalmente, do baixo custo financeiro e na redução no tempo de coleta das informações.

Há diversos estudos indicando a confiabilidade das medidas antropométricas reportadas (1-3,8,14). Nesses estudos tem sido demonstrado que os valores referidos de peso corporal por mulheres, indiferente da faixa etária tendem a serem subestimados (8), sendo superestimadas as medidas referidas de estatura (1).

O fato das medidas reportadas serem com valores abaixo do que foi aferida pode ser explicado em parte, pela maior preocupação das mulheres com o aumento do peso corporal, em que a obtenção de medidas, sejam essas aferidas ou reportadas proporcionaria constrangimento à pessoa avaliada. E essa pessoa poderá subestimar as medidas reportadas de peso corporal por uma condição de conforto pessoal. Já em relação às medidas 
de estatura referida, as mulheres em geral não possuem o hábito de realizar tal medida, e ainda, somado as mudanças morfológicas comuns ao envelhecimento, faz com que as medidas sejam superestimadas.

No entanto, houveram algumas particularidades que converge com o que é apresentado na literatura (2) como foi o caso do peso reportado, em que as idosas no melhor estrato econômico tinham a tendência de superestimar o peso corporal. Na estatura, os dados foram superestimados somente pelas pessoas com melhor escolaridade e classificadas como obesas.

Os resultados obtidos do peso corporal reportado são similares a outros estudos $(15,16)$ que revelam índices de concordância classificado como "quase perfeita", entre as medidas antropométricas aferidas com as reportadas. Porém, o mesmo não aconteceu com as medidas reportadas da estatura e IMC. Talvez tais diferenças possam ser explicadas pelo baixo nível econômico e de escolaridade da população investigada.

Em geral, as pessoas em melhores estratos econômicos e maior nível de escolaridade, possuem hábitos de vigilância à saúde, o que inclui revisão de condições de saúde periódicas e cuidados estéticos (17). Assim, parece ser mais fácil para essas pessoas reportarem com mais precisão as suas medidas, sejam elas de peso ou estatura corporal.

As medidas reportadas de peso corporal, apesar de apresentarem índices de concordância satisfatórios com as medidas aferidas, diminuem em função do aumento da faixa etária, diminuição da escolaridade e classe econômica, presença de déficit cognitivo e a participação em atividades físicas de lazer.

A diminuição do índice de concordância das medidas aferidas com as reportadas em pessoas que participam de atividades físicas de lazer parece estar relacionado ao fato que as pessoas mais ativas, tenderem a uma visão mais otimista da sua condição de saúde, o que pode ter contribuído para distorção do peso corporal real (aferido) (18).

No presente estudo, os valores das medidas antropométricas referidos tenderam a serem subestimados e, ainda, houve uma maior subestimação da estatura, quando comparada com o peso corporal. Tal fato proporcionou que o IMC fosse superestimado, exceto para as mulheres obesas, ao contrário de outros estudos $(2,16)$ que demonstram que o IMC reportado em mulheres tende para a subestimação dos valores. 
As pessoas classificadas com sobrepeso foram as mais erroneamente classificadas. Talvez pelo fato de transição no peso corporal em que essas pessoas se encontrem, faça com que a percepção da estatura e peso corporal não seja eficaz, resultando em distorções da classificação do IMC. Há estudos que recomendam cautela na aplicação de medidas referidas para cálculo do IMC, devido às distorções em função das condições de saúde e variações morfológicas (19), principalmente em pessoas mais idosas.

As medidas antropométricas referidas são frequentemente utilizadas em levantamentos populacionais $(7,20)$ devido aos estudos originais de validação das mesmas, indicarem bons níveis psicométricos $(8,14)$. Os resultados obtidos para o peso corporal referido são similares a outros estudos, que revelam índices satisfatórios de concordância entre as medidas aferidas com as reportadas em pessoas idosas $(15,16)$. Porém, o mesmo não aconteceu com as medidas referidas de estatura, em que os índices de concordância foram classificados de "pobre" a "regular".

O treinamento prévio dos entrevistadores para aferição do peso corporal afasta, em grande parte, a possibilidade de viés de aferição. Cabe, no entanto, a possibilidade de que os resultados das medidas auto-relatadas tenham alguma influência de fatores patológicos que favoreçam para mudanças morfológicas repentinas nas idosas. E ainda, a interferência de fatores culturais e sociais, como é o caso da baixa renda e escolaridade da amostra estudada.

Os resultados permitem concluir que, apesar das variações da concordância entre as medidas aferidas com as reportadas de peso corporal com o aumento da faixa etária, baixo nível de escolaridade e econômica, presença de déficit cognitivo e participação em atividades físicas de lazer, os índices se mantiveram classificados com uma condição satisfatória. Tal fato permite sugerir que a medida referida de peso corporal possa ser considerada como opção satisfatória à ser utilizada em levantamentos populacionais envolvendo pessoas idosas. Porém, o mesmo não é recomendado para medidas reportadas de estatura e estado nutricional (IMC), em populações com características similares a do presente estudo.

No entanto, há a necessidade de realização de estudos em que possa ser analisada a estabilidade das medidas referidas e, ainda, outros fatores sejam estes sócio-demográficos ou relacionados à saúde que possam interferir na efetividade da percepção do peso e estatura corporal em pessoas idosas • 
Agradecimentos: Fundação de Amparo à Pesquisa do Estado da Bahia-FAPESB.

\section{REFERÊNCIAS}

1. Fonseca MJM, Faerstein E, Chor D, Lopes CS. Validade de peso e estatura informados e índice de massa corporal: estudo pró-saúde. Rev Saúde Pública. 2004;38(3):392-8.

2. Peixoto MRG, Benício MHD, Jardim PCB. Validade do peso e da altura auto-referidos: o estudo de Goiânia. Rev Saúde Pública. 2006;40(6):1065-72.

3. Farias Junior JC. Validade das medidas auto-referidas de peso e estatura para o diagnóstico do estado nutricional de adolescentes. Rev. Bras. Saúde Matern. Infant. 2007;7 (2): 167-174.

4. Janssen I. Influence of sarcopenia on the development of physical disability: The cardiovascular health study. J Am Geriatr Soc. 2006; 54:56-62.

5. Lower GDO. Is sticky blood a treatable determinant of cognitive decline and of dementia? Age and Ageing. 2001; 30:101-103.

6. Kuo HK, Jone RN, Milberg WP, Tennstedt S, Talbot L, Morris JN, Lipsitz LA. Cognitive Function in Normal-Weight, Overweight, and Obese Older Adults: An Analysis of the Advanced Cognitive Training for Independent and Vital Elderly Cohort. J Am Geriatr Soc. 2006; 54:97-103.

7. Instituto Brasileiro de Geografia e Estatística (FIBGE). Síntese de Indicadores Sociais. Uma análise das condições de vida da população Brasileira. Rio de Janeiro: IBGE; 2007.

8. Schmidt MI, Duncan BB, Tavares M, Polanczyk CA, Pellanda L, Zimmer PM. Validity of sefreported weight: a study of urban brazilian adults. Rev Saude Pública. 1993;27(4):271-6.

9. ANEP [Internet]. Critério de Classificação Econômica Brasil 2003; Disponível em: http:// www.abep.org/codigosguias/ABEP_CCEB_2003.pdf/ Acesso em 13/01/2010.

10. Pfeiffer $E$. A short portable mental status questionnaire for the assessment of organic brain deficit in elderly patients. J Am Geriatr Soc. 1975; 23: 433-441.

11. Almeida OP, Almeida SA. Confiabilidade da versão brasileira da escala de depressão em geriatria (GDS) versão reduzida. Arq Neuropsiquiatr. 1999; 57:421-426.

12. World Health Organization. Obesity: preventing and managing the global epidemic. Report of a WHO consultation on obesity. Geneva; 1998.

13. Landis JR, Koch GG. The measurement of observer agreement for categorical data. Biometrics. 1977; 33: 159-74.

14. Chor D, Coutinho ESF, Laurenti R. Confiabilidade da informação de peso e estatura em funcionários de banco estatal. Rev Saúde Pública. 1999;33(1):16-23.

15. Stevens J, Keil JE, Waid LR, Gazes PC. Accuracy of current, 4 year, and 28-year self-reported body weight in an elderly population. Am J Epidemiol. 1990;132:1156-63.

16. Kuczmarski MF, Kuczmarski RJ, Najjar M. Effects of age on validity of self-reported height weight, and body mass index: findings from of Third National Health and Nutrition Examination Survey, 1988-1994. J Am Diet Assoc. 2001;101(1):28-34.

17. Pinheiro RS, Travassos C. Estudo da desigualdade na utilização de serviços de saúde por idosos em três regiões da cidade do Rio de Janeiro. Cad. Saúde Pública 1999; 15(3):487496.

18. Guimarães JMN, Caldas CP. A influência da atividade física nos quadros depressivos de pessoas idosas: uma revisão sistemática. Rev Bras Epidemiol. 2006; 9(4): 481-92.

19. Santillan AA, Camargo Jr CA. Body mass index and asthma among Mexican adults: the effect of using self-reported vs measured weight and height. Int $\mathrm{J}$ Obes Relat Metab Disord. 2003;27:1430-3.

20. Veloso IS, Santana VS. Impacto nutricional do programa de alimentação do trabalhador no Brasil. Rev Panam Salud Public. 2002; 11(1):24-31. 